\title{
Pressure Garment Subsystem Roadmap
}

\author{
Amy J. Ross ${ }^{1}$ \\ NASA Johnson Space Center, Houston, TX, 77058
}

\begin{abstract}
The Constellation program pressure garment subsystem (PGS) team has created a technical roadmap that communicates major technical questions and how and when the questions are being answered in support of major project milestones. The roadmap is a living document that guides the team priorities. The roadmap also communicates technical reactions to changes in project priorities and funding. This paper presents the roadmap and discusses specific roadmap elements in detail as representative examples to provide insight into the meaning and use of the roadmap.
\end{abstract}

\section{Challenges Faced in the Design of the Constellation Pressure Garment}

$\mathrm{T}$ he Constellation program had a near-term goal of carrying astronauts to the International Space Station (ISS) and returning them home, and a longer-term goal to return humans to the moon. Under the Constellation program the space suit pressure garment subsystem (PGS) was faced with three challenges.

The first challenge was that of multiple functions. The two goals of transportation to and from ISS and lunar missions required the Constellation space suit system to perform three functions: 1] launch, entry, and abort (LEA), 2] micro-gravity extra-vehicular activity (EVA), and 3] partial gravity EVA. However, spacecraft are always limited on mass and volume, so the pressure garment team was driven to design a system that provided the three functionalities in as little mass and volume of hardware as possible.

The second challenge was schedule. The Constellation program and the suit contract have a chronologic separation between the due date for missions to the ISS, which required LEA functions, and the due date for the lunar mission capability of partial gravity EVA capability. The schedule disconnect was also imposed by the space suit development contract structure which limited lunar EVA pressure garment design efforts during the initial contract phase.

The third challenge was the effective incorporation of the results from EVA technology development efforts. Technology development efforts were critical to the success of the space suit system design. This challenge also was increased because pressure garment technology development efforts were not funded until the same fiscal year in which the preliminary design review for the ISS configuration was to be performed.

\section{Approaches to Pressure Garment Design Challenges}

The programmatic approach to address the challenge of multiple functions in the lowest mass and volume of hardware as possible was to impose a modular suit system approach. The suit system would have two configurations, one primarily for LEA activities and the other for partial gravity functions, with components that to be were used in both configurations. The components that were to be used on both configurations were termed 'modular'. The proposed modular components of the Constellation pressure garment shown in Fig. 1, are colorcoded with the perceived risk to the feasibility of modularity for that component.

The modular approach was also expected to limit the impact of the schedule disconnect. Modular components had to consider lunar requirements, but during the majority of the initial configuration design, lunar requirements would not been completed. The Constellation program milestone for review and approval of top level lunar requirements did not occur until late in the design cycle of the initial configuration. Therefore, modular components had to be designed with best assumptions for lunar requirements. The risk was accepted with the consideration that

\footnotetext{
${ }^{1}$ Space Suit Engineer, Crew Survival and Space Suit Systems Branch, EC5.
} 
a beneficial aspect of the modular system is that if requirement assumptions were wrong, then only components may have to be redesigned instead of the pressure garment in its entirety.

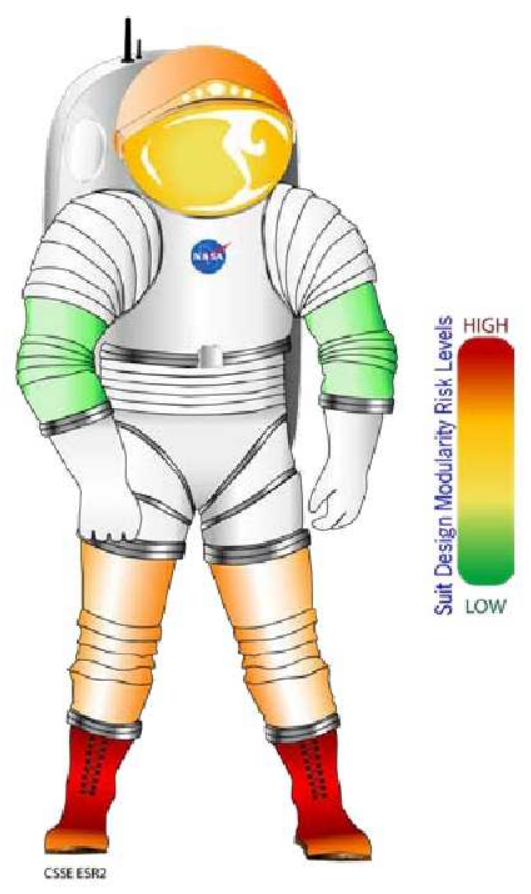

Figure 1: Modular approach to multi-function pressure garment

The modular approach, however, created some risk. Prior multi-functional pressure garment designs had not attempted to obtain such high level performance in the all three of the functional areas, which meant that multiple and often conflicting requirements, were allocated on individual modular components. Recent pressure garment designs, both flight systems and prototypes, had been specialized for LEA, micro-gravity EVA, or partial-gravity EVA capability. Specialization for a single function was based on the basic space suit design tenants that the form a pressure garment design takes depends on two key drivers: 1] the functional capability needed based on the operational concept and 2] the environmental protection required based on where the pressure garment would be used. For example, the Space Shuttle Extra-vehicular Mobility Unit (Shuttle EMU) was the first suit to be designed solely for low earth orbit micro-gravity EVA. Its form reveals its optimization for that work and environment from its boots designed for foot restraint interface, limited lower torso mobility, details of the type and placement of upper torso mobility features, and the multi-layer insulation of the thermal micrometeoroid garment. Therefore, the technical risks associated with the modular approach drove feasibility studies to determine if the modular components could be designed successfully .

In some cases, the results of a technology development effort were necessary to enable the design of modular components, making technology development critical to the Constellation pressure garment. Another aspect of the challenge of the effective incorporation of the results from technology development was that the technology development program followed a different program priorities from that of the pressure garment. This program differences compounded the previously identified programmatic schedule disconnect and adds to the risk to of technology being developed to necessary maturity level when needed for incorporation. Again, the programmatic intention was that the modular approach allowed technology to be inserted into the suit system at the component level with limited cost, schedule, and performance impacts. 


\section{Roadmaps}

\section{A. Integrated Master Schedule}

Managing the above challenges requires insight into the work being performed and how that work contributes to meeting technical goals and programmatic milestones. The prime space suit contract required that work be managed and tracked in an integrated master schedule (IMS). The integrated master schedule listed all major tasks and decomposes some tasks. The IMS is used to track project status for Earned Value Management (EVM). Listed tasks include trades, analyses, and feasibility studies. However, while dependencies and linkages are indicated in the IMS, its sheer size made it difficult to readily discern what intermediate decisions were required to be made in advance of programmatic milestones. Moreover, the IMS provides no insight into the implications of the various potential outcomes and answers made at decision points. It was this lack of logic insight and the implications of various decisions in the IMS that motivated the development of roadmaps.

\section{B. Roadmap Objectives}

Roadmaps were developed to manage the three challenges and to provide the insight into the logic behind the tasks in the IMS. The primary purpose of the roadmaps was communication. Although the roadmaps do indicate schedule at a top-level via programmatic milestones, more importantly, the roadmaps communicate key decisions, implications and flight program risk associated with decisions, and clear rationale for technology development funding requests. The balance of this paper is a discussion of the roadmaps as illustrated with the glove and EVA thermal, micrometeoroid garment (TMG) and thermal, micrometeoroid, and dust garment (TMDG) roadmaps. The glove roadmap is more representative of near-term pressure garment component design issues, while the TMG/TMDG roadmap content is more representative of a technology development-oriented design path.

While Gantt chart schedules, such as the IMS, record lists of tasks and can indicate relationships between tasks, the logic of the work flow may not be clear on large integrated schedules. The roadmaps indicate when, in relation to project milestones, it is necessary for major decisions to be made. In addition to identifying what major design decisions needed to be made and indicating when with respect to project milestones, the roadmaps were developed to communicate the following:

- what information was feeding the decision milestones

- the implications of decisions

- when technology development results could be incorporated into the flight program design effort, and

- from what funding source various efforts were being supported.

\section{Roadmap Format}

Prior to the discussion of the roadmaps, it will be helpful to review the format and information included. A roadmap was developed for each PGS contract end item (CEI), and the roadmap is labeled, as seen in Fig.s 2 and 3, with the CEI title on the top left-hand corner. A CEI is a distinct PGS component, such as the helmet, liquid cooling and ventilation garment, or gloves. The top of each roadmap is labeled with project milestones. The milestones are delineated by name and not date for two reasons. First, the logic of the design is based on logic flow of the design process, so the design milestones are appropriate decision point. Second, it was more efficient to use the names instead of dates due to the fluidity experienced in the project schedule. The milestones are weighted toward ISS mission milestones, with inclusion of the PGS CEI preliminary design review (PDR), labeled as "CEI PDR"; Constellation Suit System Element (CSSE) PDR, labeled as "ISS Config PDR"; and CSSE interim design review, labeled as "ISS Config IDR", which is the review that approves a design for design verification testing. The lunar mission milestones of lunar EVA system requirements review (lunar SRR) and lunar configuration PDR milestones are separated by a notional milestone for achieving technology readiness level (TRL) 6 . TRL 6 represents a stage of technology development at which it is feasible to include the technology into flight system hardware design. The TRL milestone is an important decision point at which technology development efforts must be evaluated.

Decisions are indicated with diamonds. The decision diamonds are the core of the roadmap. They show what issues need to be resolved by when. This is the logic behind the list of tasks in the IMS. The questions tied to each 
of the design decision diamonds are major design questions that motivate efforts, such as trade studies, analyses, mock-up fabrication, testing, and feasibility studies, to provide data so that the questions can be answered. Additionally, program risks, which are shown in ovals, also drive tasks to provide data to the decisions, because often tasks and decision points on the roadmap are a part of the risk mitigation plan associated with the risk. Finally, to the right of the decision diamonds the various implications of each potential answer is listed. By this means, the roadmaps communicate valuable planning information of cost, schedule and technical impacts of the decisions being made. Early top level insight of this kind provides the opportunity for proactive planning instead of having to react to decision repercussions at milestones. The foresight granted by the roadmaps grant enhances the value of the tool.

The final information illustrated on the roadmap is that of funding sources. The legend is located in the bottom left-hand corner. The EVA Systems Project Office (ESPO) funded all Constellation-related efforts. The majority of that funding was allocated to the space suit prime contractor. However, there are two paths of funding to the space suit prime contractor: the base contract and the "indefinite delivery, indefinite quantity" (IDIQ) mechanism. The IDIQ enables special tasks to be funded to supplement base contract scope. "ESPO direct" labeled funding indicates IDIQ or other efforts funded by the ESPO. Efforts funded by the Exploration Technology Development Program (ETDP) are indicated in green. Other sources of funding refers to any funding source outside of the ESPO and ETDP. Examples include division and center discretionary funds, small business innovative research grants, and Space Human Factors Engineering. Finally, work that made sense to include on the roadmap, but did not have an identified funding source, was labeled in red, which highlights funding needs.

\section{Glove Roadmap}

The glove roadmap concentrates on the scope of work necessary to provide gloves required for the ISS mission. Shown as concurrent on the far left are a life cycle cost trade and new glove internal development. The tasks are specified by the task description number used by the space suit prime contractor in the IMS that refers to the task description sheet (TDS), which scopes the effort. One objective of the life cycle cost trade was an analysis to determine if a current glove configurations could meet Constellation requirements, both ISS and lunar, or if a new glove development would be required. Ideally, this trade would be completed first and then effort expended on new glove development only if it were required. Therefore, the roadmap indicates that the schedule available prior to CEI PDR was limited to the degree that the two efforts could not be performed serially. The decision diamond to determine if a new glove would be required was fed by the two efforts and had to be answered before CEI PDR so that a single approach could be presented at CEI PDR. The roadmap communicates three possible answers. It is helpful to understand the possible outcomes. For example, over the year prior to CEI PDR, EVA coordinated with the Orion project to provide hardware for vehicle interface tests. Based on the roadmap, the PGS team understood the range of configurations that are plausible to test and communicated that with Orion. Additionally, the different glove approaches, specifically a new glove development versus production or modification of the current Shuttle space suit Phase VI glove, represent various levels of effort by different members of the prime contractor's team. Awareness of the options aids in preparing for any of the answers. The next decision diamond, placed prior to the ISS configuration PDR, reviews the decision made at CEI PDR. This later decision is fed by mock-up test result shown to result from the internal glove development effort, if that path is pursued.

Although the glove roadmap emphasizes ISS capability design, current efforts funded by the ETDP for longduration gloves are indicated at the bottom of the roadmap. ETDP funded testing to determine the baseline durability performance of Phase VI gloves that have been exposed to lunar stimulant. The test will demonstrate the gap between current durability capability and that required for exploration missions, which then serves as rationale for future funding requests and a mark from which to measure progress. The roadmap also indicates that future glove work has not yet been funded. 


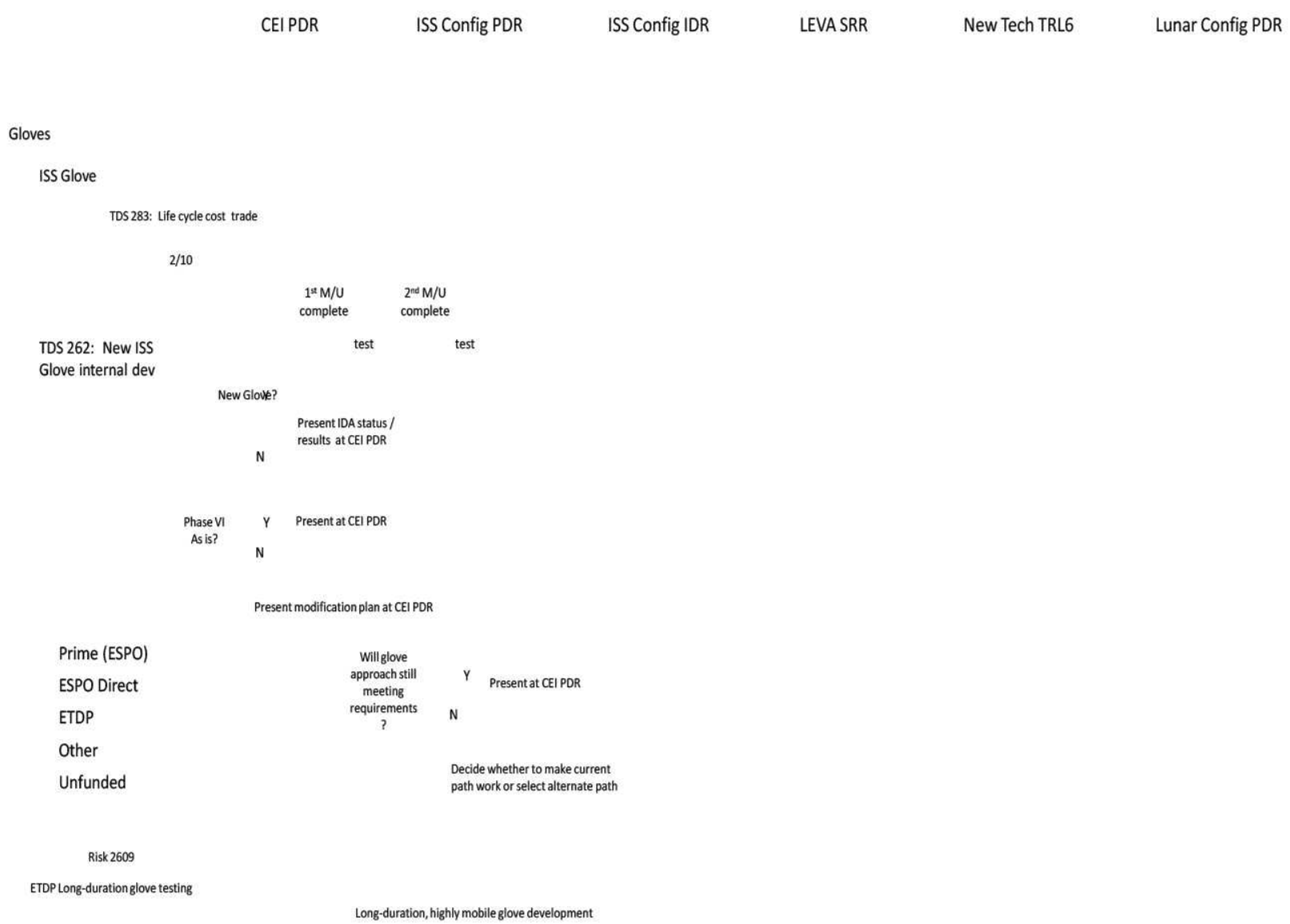

Figure 2: Glove Roadmap

\section{E. Thermal, Micrometeoroid Garment and Thermal, Micrometeoroid and Dust Garment}

The TMG/TMDG roadmap, while addressing near-term requirements, focuses detailed planning on technology development for exploration mission. This is true because, in contrast to the glove situation, the perceived risk of current flight system hardware being able to meet the Constellation requirements was low. Again, the roadmap communicates the specific tasks being performed by the space suit prime contractor for the TMG. The logic shown is that the TMG material lay-up is defined by CEI PDR. The specific architecture of the TMG, meaning how many pieces there are of TMG and where and how they are connected to the pressure garment, is planned to be determined during the detailed design phase following ISS configuration PDR. The logic here is that the segmentation of the TMG is driven the final space suit configuration approved at ISS configuration PDR. The segmentation architecture is verified via prototyping prior to design verification testing, per the decision diamond at the ISS configuration IDR milestone.

The roadmap indicates that a task has been completed and another is on-going for TMDG development, but the majority of the effort is in out years. The TMDG work is divided into outer layer and multi-layer insulation (MLI) efforts. This is meaningful. It indicates a two-prong approach to dust resistance. The first is to use the outer layer as a means to keep dust from penetrating deeper. The MLI task investigates the performance of dirty and worn materials. In other words, as the MLI degrades over time on what relative curve does the thermal performance degrade. Both the outer layer and MLI tasks leave room in the schedule for technology development of new materials to TRL 6, if current technologies cannot meet exploration requirements. The roadmap also indicates that thermal performance is a critical design criteria for the outer layer. Finally, the roadmap communicates that the individual layers, when combined, must meet the combined performance requirements. The decision diamond for 
TMDG performance precedes the TRL 6 milestone. By clearly showing the near-term flight program work and the logic flow and schedule drivers for the long-term technology development efforts, the TMG/TMDG roadmap well illustrates the value of the roadmap as a communication tool.

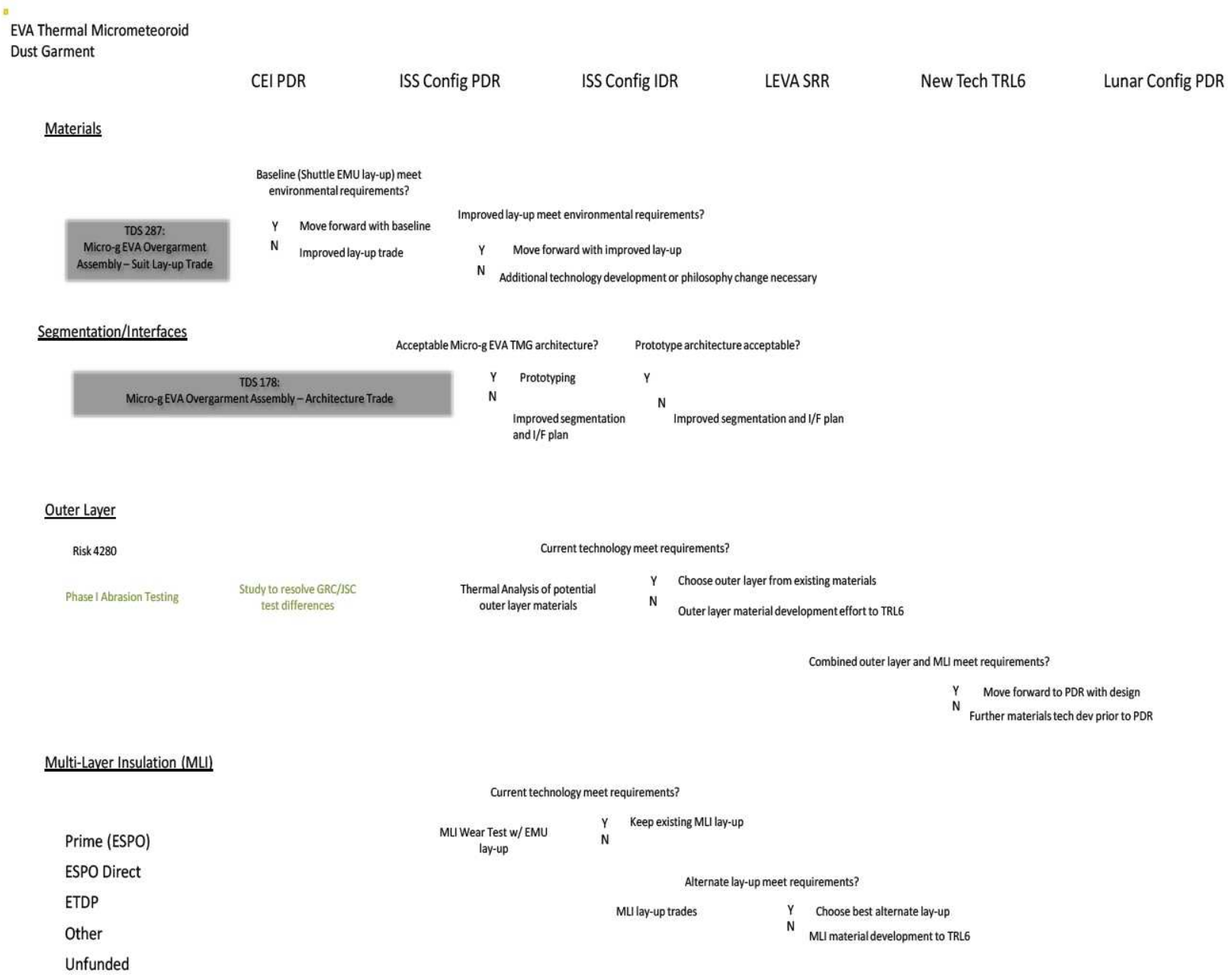

\section{Application of Roadmaps Beyond Constellation}

With the announced cancellation of the Constellation Program, NASA is working to define a new paradigm. In the current environment of redirection and adjustment, the need to communicate has only increased. The roadmaps had already considered lunar development and technology development tasks and, as living documents, the roadmaps can be reworked for any project philosophy and timeline. In the end the ability to communicate in a clear, logical and succinct format is always helpful when gaining consensus on a technical plan, providing rationale for funding requests, and managing space suit development leveraged across projects and programs, activities in which the space suit team is continuously engaged.

\section{Acknowledgments}

The author would like to thank Kate Mitchell, Mary Hakam, Shawn Macleod, and Don Tufts for their inputs toward the development of the TMG/TMDG and glove roadmaps. 